\title{
Analytical Hartree-Fock gradients for periodic systems
}

\author{
K. Doll ${ }^{1}$, V. R. Saunders ${ }^{1}$ and N. M. Harrison ${ }^{1,2}$ \\ ${ }^{1}$ CLRC, Daresbury Laboratory, Daresbury, Warrington, WA4 4AD, UK \\ ${ }^{2}$ Department of Chemistry, Imperial College, London, SW7 $2 A Y, U K$
}

\begin{abstract}
We present the theory of analytical Hartree-Fock gradients for periodic systems as implemented in the code CRYSTAL. We demonstrate how derivatives of the integrals can be computed with the McMurchie-Davidson algorithm. Highly accurate gradients with respect to nuclear coordinates are obtained for systems periodic in $0,1,2$ or 3 dimensions.
\end{abstract}

Typeset using REVTEX 


\section{INTRODUCTION}

The determination of equilibrium structure is one of the most important targets in electronic structure calculations. Analytical gradients provide an important tool to facilitate this and therefore the implementation of analytical gradients has become an important part of modern codes. Although most solid state calculations are nowadays performed within the framework of density functional theory, Hartree-Fock theory can serve as a useful starting point for a correlation treatment. In the field of quantum chemistry, a Hartree-Fock solution is necessary to make a wavefunction based correlation scheme such as, for example, the coupled-cluster approach, applicable. Therefore, the determination of a Hartree-Fock solution is often an important target.

The calculation of Hartree-Fock gradients was pioneered by Pulay who performed the first implementation for multicenter basis sets1. It should be mentioned, that the theory had already been derived earlier independently日. Analytical gradients have become an important area in quantum chemistry and several review articles have been published 3 - 6 .

Significant work has already been performed for one-dimensional systems: formulas for analytic gradients, with respect to nuclear coordinates as well as with respect to the lattice vector, have been derived and implemented in a periodic code 7 ; and the theory has been extended to metallic systems 8 . Further progress has been the derivation and implementation of formulas for MP2 energy 10 and gradients 11 , as well as gradients on the density functional level12. Even formulas for second derivatives have meanwhile been coded 13 . Recently, a scheme for an accurate treatment of long-range Coulombic effects in Hartree-Fock gradients has been presented 14 , and a new implementation of density functional energy and gradients for periodic systems has been demonstated to be highly efficient and accurate 15 .

In this article, we report on an implementation of Hartree-Fock gradients with respect to nuclear coordinates in a general periodic code (the CRYSTAL 19 package), which is to the best of our knowledge the first implementation for the case of 2- and 3- dimensional periodicity. 
The article is structured as follows: in section [1], the basis functions and Hartree-Fock equations are given. The calculation of integrals which relies on the McMurchie-Davidson algorithm and the calculation of gradients of the integrals is explained in sections III and IV. The total energy as calculated by the CRYSTAL code is given in section $\nabla$. In section VI, we explain the calculation of forces and possible sources of error. Finally, in section $\nabla T$ we illustrate the accuracy of the gradients with some examples.

\section{BASIS FUNCTION AND HARTREE-FOCK EQUATIONS}

In this section, we summarize the basis functions used in the CRYSTAL code and give the structure of the Hartree-Fock equations.

\section{A. Basis functions}

Unnormalized spherical Gaussian type functions (SGTF) in a polar coordinate system characterized by the set of variables $(|\vec{r}|, \vartheta, \varphi)$, and centered at $\vec{A}$, are defined as

$$
S(\alpha, \vec{r}-\vec{A}, n, l, m)=|\vec{r}-\vec{A}|^{2 n+l} \mathrm{P}_{l}^{|m|}(\cos \vartheta) \exp (\operatorname{im} \varphi) \exp \left(-\alpha|\vec{r}-\vec{A}|^{2}\right)
$$

with $\mathrm{P}_{l}^{|m|}$ being the associated Legendre function. In the context of the McMurchieDavidson algorithm, Hermite Gaussian type functions are necessary which are defined as:

$$
\Lambda(\gamma, \vec{r}-\vec{A}, t, u, v)=\left(\frac{\partial}{\partial A_{x}}\right)^{t}\left(\frac{\partial}{\partial A_{y}}\right)^{u}\left(\frac{\partial}{\partial A_{z}}\right)^{v} \exp \left(-\gamma|\vec{r}-\vec{A}|^{2}\right)
$$

Real spherical Gaussian type functions are defined as

$$
\begin{array}{r}
R(\alpha, \vec{r}-\vec{A}, n, l, 0)=S(\alpha, \vec{r}-\vec{A}, n, l, 0) \\
R(\alpha, \vec{r}-\vec{A}, n, l,|m|)=\operatorname{Re} S(\alpha, \vec{r}-\vec{A}, n, l,|m|) \\
R(\alpha, \vec{r}-\vec{A}, n, l,-|m|)=\operatorname{Im} S(\alpha, \vec{r}-\vec{A}, n, l,|m|)
\end{array}
$$

CRYSTAL uses real spherical Gaussian type functions, which are in the following denoted as $\phi_{\mu}\left(\vec{r}-\vec{A}_{\mu}\right)=N_{\mu} R\left(\alpha, \vec{A}_{\mu}, n, l, m\right)$, with the normalization $N_{\mu} \cdot \mu$ is an index enumerating 
the basis functions in the reference cell (e.g. the primitive unit cell). Although the code allows only the use of SGTFs with $n=0$, in the process of the evaluation of molecular integrals, SGTFs with $n \neq 0$ are used20.

\section{B. Hartree-Fock equations}

The Hartree-Fock treatment of periodic system\$21 is briefly repeated in this section. We assume that orbitals are doubly occupied and work within the restricted Hartree-Fock formalism. The crystalline orbitals are linear combinations of Bloch functions

$$
\Psi_{i}(\vec{r}, \vec{k})=\sum_{\mu} a_{\mu i}(\vec{k}) \psi_{\mu}(\vec{r}, \vec{k})
$$

which are expanded in terms of real spherical Gaussian type functions with fixed contraction coefficients $d_{j}$ :

$$
\psi_{\mu}(\vec{r}, \vec{k})=N_{\mu} \sum_{\vec{g}} \sum_{j} d_{j} R\left(\alpha_{j}, \vec{r}-\vec{A}_{\mu}-\vec{g}, n, l, m\right) \mathrm{e}^{\mathrm{i} \vec{k} \vec{g}}
$$

The sum over $\vec{g}$ is over all direct lattice vectors. The Hartree-Fock-Roothaan equations have a structure similar to the molecular case and have to be solved on a set of points in the reciprocal lattice:

$$
\sum_{\nu} F_{\mu \nu}(\vec{k}) a_{\nu i}(\vec{k})=\sum_{\nu} S_{\mu \nu}(\vec{k}) a_{\nu i}(\vec{k}) \epsilon_{i}(\vec{k})
$$

The Fock matrix $F$ is given in detail in section $\square$ of this article, the overlap matrix $S$ in section [II. The spin-free density matrix in reciprocal space is defined as

$$
P_{\mu \nu}(\vec{k})=2 \sum_{i} a_{\mu i}(\vec{k}) a_{\nu i}^{*}(\vec{k}) \Theta\left(\epsilon_{F}-\epsilon_{i}(\vec{k})\right)
$$

with the Fermi energy $\epsilon_{F}$ and the Heaviside function $\Theta ; i$ is an index enumerating the eigenvalues. The density matrix in real space $P_{\mu \overrightarrow{0} \nu \vec{g}}$ is obtained by Fourier transformation. 


\section{CALCULATION OF INTEGRALS}

\section{A. Types of integrals}

In this section we summarize the appearing types of integrals. This is done with the assumption that the basis functions, $\phi_{\mu}$, are real.

\section{Overlap integral}

The basic integral is the overlap integral:

$$
S_{\mu \overrightarrow{g_{1}} \nu \overrightarrow{g_{2}}}=\int \phi_{\mu}\left(\vec{r}-\overrightarrow{A_{\mu}}-\vec{g}_{1}\right) \phi_{\nu}\left(\vec{r}-\overrightarrow{A_{\nu}}-\vec{g}_{2}\right) \mathrm{d}^{3} \mathrm{r}
$$

The integration is over the whole space, i.e. $x$ from $-\infty$ to $+\infty$ and similarly for $y$ and z. Exploiting translational invariance we can rewrite this as

$$
S_{\mu \overrightarrow{0} \nu \vec{g}}=\int \phi_{\mu}\left(\vec{r}-\vec{A}_{\mu}\right) \phi_{\nu}\left(\vec{r}-\vec{A}_{\nu}-\vec{g}\right) \mathrm{d}^{3} \mathrm{r}
$$

with $\vec{g}=\overrightarrow{g_{2}}-\overrightarrow{g_{1}}$.

Further integrals appearing are:

2. Kinetic energy integrals

$$
T_{\mu \overrightarrow{0} \nu \vec{g}}=\int \phi_{\mu}\left(\vec{r}-\vec{A}_{\mu}\right)\left(-\frac{1}{2} \Delta_{\vec{r}}\right) \phi_{\nu}\left(\vec{r}-\vec{A}_{\nu}-\vec{g}\right) \mathrm{d}^{3} \mathrm{r}
$$

\section{Nuclear attraction integrals}

$$
N_{\mu \overrightarrow{0} \nu \vec{g}}=-\sum_{a} Z_{a} \int \phi_{\mu}\left(\vec{r}-\vec{A}_{\mu}\right) A\left(\vec{r}-\vec{A}_{a}\right) \phi_{\nu}\left(\vec{r}-\vec{A}_{\nu}-\vec{g}\right) \mathrm{d}^{3} \mathrm{r}
$$

where $A$ is defined as the Coulomb potential in the molecular case, as the EulerMacLaurin potential for systems periodic in one dimension22,23, as Parry's potential24 for systems periodic in two dimensions, and as the Ewald potential for systems periodic in three dimensions 25,26. The a summation runs over all nuclei of the primitive unit cell. 


\section{Electron-electron Coulomb interaction integrals}

A bielectronic integral can be defined as

$$
B_{\mu \overrightarrow{0} \nu \vec{g} \tau \vec{n} \sigma \vec{n}+\vec{h}}=\int \frac{\phi_{\mu}\left(\vec{r}-\vec{A}_{\mu}\right) \phi_{\nu}\left(\vec{r}-\vec{A}_{\nu}-\vec{g}\right) \phi_{\tau}\left(\vec{r}^{\prime}-\vec{A}_{\tau}-\vec{n}\right) \phi_{\sigma}\left(\vec{r}^{\prime}-\vec{A}_{\sigma}-\vec{n}-\vec{h}\right)}{\left|\vec{r}-\vec{r}^{\prime}\right|} \mathrm{d}^{3} \mathrm{r}^{3} \mathrm{r}^{\prime}
$$

In the context of periodic systems, it is necessary to perform summation over all lattice vectors $\vec{g}, \vec{h}, \vec{n}$. We define a Coulomb integral as follows

$$
\begin{aligned}
& C_{\mu \overrightarrow{0} \nu \vec{g} \tau \overrightarrow{0} \sigma \vec{h}}=\sum_{\vec{n}}^{p e n} \int \frac{\phi_{\mu}\left(\vec{r}-\vec{A}_{\mu}\right) \phi_{\nu}\left(\vec{r}-\vec{A}_{\nu}-\vec{g}\right) \phi_{\tau}\left(\vec{r}^{\prime}-\vec{A}_{\tau}-\vec{n}\right) \phi_{\sigma}\left(\vec{r}^{\prime}-\vec{A}_{\sigma}-\vec{n}-\vec{h}\right)}{\left|\vec{r}-\vec{r}^{\prime}\right|} \mathrm{d}^{3} \mathrm{rd}^{3} \mathrm{r}^{\prime}= \\
& \sum_{\vec{n}}^{p e n} B_{\mu \overrightarrow{0} \nu \vec{g} \tau \vec{n} \sigma \vec{n}+\vec{h}}
\end{aligned}
$$

The penetration depth pen is defined as those terms for which

$$
\int\left[g\left(\alpha_{\mu}^{m i n}, \vec{r}-\vec{A}_{\mu}\right) g\left(\alpha_{\nu}^{m i n}, \vec{r}-\vec{A}_{\nu}-\vec{g}\right)\right]^{1 / 2} g\left(\alpha_{\tau}^{m i n}, \vec{r}-\vec{A}_{\tau}-\vec{n}\right) \mathrm{d}^{3} \mathrm{r}>10^{-I T O L 2}
$$

holds, with $g\left(\alpha_{a}^{\min }, \vec{r}\right)=\left(\frac{2 \alpha_{a}^{\min }}{\pi}\right)^{3 / 4} \exp \left(-\alpha_{a}^{\min }|\vec{r}|^{2}\right)$.

$g\left(\alpha_{a}^{\min }, \vec{r}\right)$ means the lowest exponent of all Gaussians centered at $\vec{A}_{a}$. For these integrals, the Coulomb interaction is evaluated without approximation. All the other integrals are evaluated with a multipolar expansion. "ITOL2" is a tolerance which can be chosen by the user of the code. This criterion introduces an asymmetry in the energy expression: a given bielectronic integral might be evaluated in different ways for $B_{\mu \overrightarrow{0} \nu \vec{g} \tau \vec{n} \sigma \vec{n}+\vec{h}}$ and $B_{\tau \vec{n} \sigma \vec{n}+\vec{h} \mu \overrightarrow{0} \nu \vec{g}}$. Avoiding this would however be very inefficient: To keep the symmetry, the lattice sum would have to be further broken down into pieces which are evaluated exactly and other pieces which are approximated — this would require a much higher computational effort and more disk storage. The simpler criterion in equation 13 minimizes the effort, and, when ITOL2 is chosen sufficiently large, the violation of the symmetry is negligible. We will illustrate this with some examples in section $\nabla 11$. 


\section{Electron-electron exchange interaction integrals}

$$
\begin{aligned}
& X_{\mu \overrightarrow{0} \nu \vec{g} \tau \overrightarrow{0} \sigma \vec{h}}=\sum_{\vec{n}} \int \frac{\phi_{\mu}\left(\vec{r}-\vec{A}_{\mu}\right) \phi_{\tau}\left(\vec{r}-\vec{A}_{\tau}-\vec{n}\right) \phi_{\nu}\left(\vec{r}^{\prime}-\vec{A}_{\nu}-\vec{g}\right) \phi_{\sigma}\left(\vec{r}^{\prime}-\vec{A}_{\sigma}-\vec{n}-\vec{h}\right)}{\left|\vec{r}-\vec{r}^{\prime}\right|} \mathrm{d}^{3} \mathrm{rd}^{3} \mathrm{r}^{\prime}= \\
& \sum_{\vec{n}} B_{\mu \overrightarrow{0} \tau \vec{n} \nu \vec{g} \sigma \vec{n}+\vec{h}}
\end{aligned}
$$

For an individual exchange integral,

$$
B_{\mu \overrightarrow{0} \tau \vec{n} \nu \vec{g} \sigma \vec{n}+\vec{h}}=B_{\tau \vec{n} \mu \overrightarrow{0} \sigma \vec{n}+\vec{h} \nu \vec{g}}
$$

should hold. However, for efficiency reasons, two different thresholds have been introduced 16 which leads to another possible asymmetry: an exchange integral is discarded when the pseudooverlap associated with $\phi_{\mu}\left(\vec{r}-\vec{A}_{\mu}\right)$ and $\phi_{\nu}\left(\vec{r}-\vec{A}_{\nu}-\vec{g}\right)$ or the pseudooverlap associated with $\phi_{\tau}\left(\vec{r}-\vec{A}_{\tau}-\vec{n}\right)$ and $\phi_{\sigma}\left(\vec{r}-\vec{A}_{\sigma}-\vec{n}-\vec{h}\right)$ is smaller than certain thresholds, $10^{-I T O L 4}$ and $10^{-I T O L 5}$. It is recommended that the threshold ITOL5 associated with

$\phi_{\tau}\left(\vec{r}-\vec{A}_{\tau}-\vec{n}\right)$ and $\phi_{\sigma}\left(\vec{r}-\vec{A}_{\sigma}-\vec{n}-\vec{h}\right)$ should be higher than ITOL4. This, however, will lead to a violation of equation 15 and therefore another asymmetry in the energy expression. A further cutoff parameter, ITOL3, selects the exchange integrals symmetrically: exchange integrals are also neglected if the overlap of $\phi_{\mu}\left(\vec{r}-\vec{A}_{\mu}\right)$ with $\phi_{\tau}\left(\vec{r}-\vec{A}_{\tau}-\vec{n}\right)$ or the overlap of $\phi_{\nu}\left(\vec{r}-\vec{A}_{\nu}-\vec{g}\right)$ with $\phi_{\sigma}\left(\vec{r}-\vec{A}_{\sigma}-\vec{n}-\vec{h}\right)$ is lower as $10^{-I T O L 3}$. This is a symmetric cutoff, and therefore should not lead to an inaccuracy in the forces.

\section{Multipolar integrals}

The charge distribution is approximated with the help of a multipolar expansion up to order $L$.

$$
\eta_{l}^{m}\left(\rho ; \vec{A}_{c}\right)=\int \rho(\vec{r}) X_{l}^{m}\left(\vec{r}-\vec{A}_{c}\right) \mathrm{d}^{3} \mathrm{r}
$$

with $X_{l}^{m}$ being regular solid harmonic 26 and the charge $\rho(\vec{r})$ defined as

$$
\rho(\vec{r})=-\sum_{\vec{g}, \mu, \nu} P_{\nu \vec{g} \mu \overrightarrow{0}} \phi_{\mu}\left(\vec{r}-\vec{A}_{\mu}\right) \phi_{\nu}\left(\vec{r}-\vec{A}_{\nu}-\vec{g}\right)
$$




\section{Field integrals}

The electrostatic potential is approximated with an expansion up to the maximum quantum number $L$.

$$
M_{l \mu \overrightarrow{0} \nu \vec{g} c}^{m}=Z_{l}^{m}\left(\hat{\overrightarrow{A_{c}}}\right) \int \phi_{\mu}\left(\vec{r}-\vec{A}_{\mu}\right) \phi_{\nu}\left(\vec{r}-\vec{A}_{\nu}-\vec{g}\right)\left[A\left(\vec{r}-\vec{A}_{c}\right)-\sum_{\vec{n}}^{p e n} \frac{1}{\left|\vec{r}-\vec{A}_{c}-\vec{n}\right|}\right] \mathrm{d}^{3} \mathrm{r}
$$

with $Z_{l}^{m}\left(\hat{\overrightarrow{A_{c}}}\right)$ being the spherical gradient operator, $Z_{l}^{m}\left(\hat{\overrightarrow{A_{c}}}\right)=\frac{N_{l}^{m}}{(2 l-1) ! !} X_{l}^{m}\left(\hat{\overrightarrow{A_{c}}}\right)$ and a normalization $N_{l}^{m}$ (Ref. 26).

\section{Spheropole}

This term arises because the charge distribution is approximated by a model charge distribution in the long range. However, the use of the Ewald potential instead of the Coulomb potential requires a correction in the three-dimensional case 26 .

$$
Q=\sum_{c} Q_{c}=\sum_{c} \frac{2 \pi}{3 V} \int\left(\rho_{c}(\vec{r})-\rho_{c}^{\operatorname{model}}(\vec{r})\right)|\vec{r}|^{2} \mathrm{~d}^{3} \mathrm{r}
$$

with

$$
\rho_{c}(\vec{r})=-\sum_{\mu \in c} \sum_{\vec{g}, \nu} P_{\nu \vec{g} \mu \overrightarrow{0}} \phi_{\mu}\left(\vec{r}-\overrightarrow{A_{\mu}}\right) \phi_{\nu}\left(\vec{r}-\vec{A}_{\nu}-\vec{g}\right)
$$

and

$$
\rho_{c}^{\text {model }}(\vec{r})=\sum_{l=0}^{L} \sum_{m=-l}^{l} \eta_{l}^{m}\left(\rho_{c} ; \overrightarrow{A_{c}}\right) \delta_{l}^{m}\left(\vec{A}_{c}, \vec{r}\right)
$$

and

$$
\delta_{l}^{m}\left(\overrightarrow{A_{c}}, \vec{r}\right)=\lim _{\alpha \rightarrow \infty} Z_{l}^{m}\left(\hat{\overrightarrow{A_{c}}}\right) \Lambda\left(\alpha, \vec{r}-\vec{A}_{c}, 0,0,0\right)
$$

$c$ is chosen as the set of basis functions sited at center $\vec{A}_{c}$. 


\section{B. McMurchie-Davidson algorithm}

In this section, we indicate how the integrals are evaluated. This is done with the McMurchie-Davidson27 algorithm. In this formalism, the product of two Gaussian type functions is expanded at an intermediate center in terms of Hermite Gaussian type functions.

$$
\begin{array}{r}
S(\alpha, \vec{r}-\vec{A}, n, l, m) S(\beta, \vec{r}-\vec{B}, \tilde{n}, \tilde{l}, \tilde{m})= \\
\sum_{t, u, v} E(n, l, m, \tilde{n}, \tilde{l}, \tilde{m}, t, u, v) \Lambda(\gamma, \vec{r}-\vec{P}, t, u, v)
\end{array}
$$

with $\gamma=\alpha+\beta$ and $\vec{P}=\frac{\alpha \vec{A}+\beta \vec{B}}{\alpha+\beta}$. E also depends on $\alpha, \beta$ and the distance $\vec{B}-\vec{A}$; however, the dependence on these parameters is suppressed in the notation.

This makes a very efficient evaluation of integrals feasible. The starting point $E(0,0,0,0,0,0,0,0,0)=\exp \left(-\frac{\alpha \beta}{\alpha+\beta}|\vec{B}-\vec{A}|^{2}\right)$ can be derived from the Gaussian product ruld2829:

$$
\exp \left(-\alpha|\vec{r}-\vec{A}|^{2}\right) \exp \left(-\beta|\vec{r}-\vec{B}|^{2}\right)=\exp \left(-\frac{\alpha \beta}{\alpha+\beta}|\vec{B}-\vec{A}|^{2}\right) \exp \left(-(\alpha+\beta)\left|\vec{r}-\frac{\alpha \vec{A}+\beta \vec{B}}{\alpha+\beta}\right|^{2}\right)
$$

The coefficients $E$ can be generated by recursion relations 2720 . They are zero for the case $t+u+v>2 n+2 \tilde{n}+l+\tilde{l}$ and for all negative values of $t, u$ or $v$.

\section{CALCULATION OF DERIVATIVES}

\section{A. Gradients within the McMurchie-Davidson algorithm}

The evaluation of gradients of the integrals is closely related to the evaluation of the integrals themselves. All the integrals can be expressed with the help of the $E$ coefficients2 20263. In the following we show how derivatives of the integrals can be expressed in a similar way with Hermite Gaussian type functions. Starting from equation 23, we obtain 


$$
\begin{aligned}
& \frac{\partial}{\partial A_{x}}(S(\alpha, \vec{r}-\vec{A}, n, l, m) S(\beta, \vec{r}-\vec{B}, \tilde{n}, \tilde{l}, \tilde{m}))= \\
& \frac{\partial}{\partial A_{x}} \sum_{t, u, v} E(n, l, m, \tilde{n}, \tilde{l}, \tilde{m}, t, u, v) \Lambda(\gamma, \vec{r}-\vec{P}, t, u, v)= \\
& \sum_{t, u, v}\left(\left(\frac{\partial}{\partial A_{x}} E(n, l, m, \tilde{n}, \tilde{l}, \tilde{m}, t, u, v)\right) \Lambda(\gamma, \vec{r}-\vec{P}, t, u, v)+\right. \\
& \left.\frac{\alpha}{\alpha+\beta} E(n, l, m, \tilde{n}, \tilde{l}, \tilde{m}, t, u, v) \Lambda(\gamma, \vec{r}-\vec{P}, t+1, u, v)\right)= \\
& \left.\sum_{t, u, v}\left(\frac{\partial}{\partial A_{x}} E(n, l, m, \tilde{n}, \tilde{l}, \tilde{m}, t, u, v)+\frac{\alpha}{\alpha+\beta} E(n, l, m, \tilde{n}, \tilde{l}, \tilde{m}, t-1, u, v)\right) \Lambda(\gamma, \vec{r}-\vec{P}, t, u, v)\right)= \\
& \sum_{t, u, v} G_{x}^{A}(n, l, m, \tilde{n}, \tilde{l}, \tilde{m}, t, u, v) \Lambda(\gamma, \vec{r}-\vec{P}, t, u, v)
\end{aligned}
$$

Therefore, the gradients can be obtained in a quite similar way as the integrals. Instead of the $E$-coefficients, the coefficients $G_{x}^{A}$ (and, for the other derivatives, $G_{y}^{A}, G_{z}^{A}, G_{x}^{B}, G_{y}^{B}$ and $G_{z}^{B}$ ) have to be used, as defined in equation 25. We obtain the following relation from equation 25:

$$
\begin{array}{r}
G_{x}^{A}(n, l, m, \tilde{n}, \tilde{l}, \tilde{m}, t, u, v)= \\
\frac{\partial}{\partial A_{x}} E(n, l, m, \tilde{n}, \tilde{l}, \tilde{m}, t, u, v)+ \\
\frac{\alpha}{\alpha+\beta} E(n, l, m, \tilde{n}, \tilde{l}, \tilde{m}, t-1, u, v)
\end{array}
$$

We could thus derive the $G$-coefficients from the $E$-coefficients. However, a more convenient way would be to have a recursion relation similar to the E-coefficients. Indeed, these relations can be obtained in an analogous way ${ }^{19}$. We give the relations here for the case of complex spherical Gaussian type functions; a transformation to real spherical Gaussian type functions is possible along the lines given in ref. 18. The starting point can be obtained from equation 26 and the definition of $E(0,0,0,0,0,0,0,0,0)$ given in section [II B:

$$
\begin{array}{r}
G_{x}^{A}(0,0,0,0,0,0,0,0,0)= \\
\frac{\partial}{\partial A_{x}} E(0,0,0,0,0,0,0,0,0)+ \\
\frac{\alpha}{\alpha+\beta} E(0,0,0,0,0,0,-1,0,0)= \\
2 \frac{\alpha \beta}{\alpha+\beta}\left(B_{x}-A_{x}\right) E(0,0,0,0,0,0,0,0,0)
\end{array}
$$


and

$$
\begin{array}{r}
G_{x}^{A}(0,0,0,0,0,0,1,0,0)= \\
\frac{\partial}{\partial A_{x}} E(0,0,0,0,0,0,1,0,0)+ \\
\frac{\alpha}{\alpha+\beta} E(0,0,0,0,0,0,0,0,0)= \\
\frac{\alpha}{\alpha+\beta} E(0,0,0,0,0,0,0,0,0)
\end{array}
$$

All the other $G_{x}^{A}(0,0,0,0,0,0, t, u, v)$ are zero. Similarly, we obtain

$$
\begin{array}{r}
G_{y}^{A}(0,0,0,0,0,0,0,1,0)= \\
G_{z}^{A}(0,0,0,0,0,0,0,0,1)= \\
G_{x}^{A}(0,0,0,0,0,0,1,0,0)
\end{array}
$$

and

$$
\begin{aligned}
& G_{y}^{A}(0,0,0,0,0,0,0,0,0)= \\
& 2 \frac{\alpha \beta}{\alpha+\beta}\left(B_{y}-A_{y}\right) E(0,0,0,0,0,0,0,0,0) \\
& \text { and }
\end{aligned}
$$

$$
\begin{array}{r}
G_{z}^{A}(0,0,0,0,0,0,0,0,0)= \\
2 \frac{\alpha \beta}{\alpha+\beta}\left(B_{z}-A_{z}\right) E(0,0,0,0,0,0,0,0,0)
\end{array}
$$

Recursion relations for the $G$-coefficients can be derived using similar arguments as for the $E$-coefficients $\sqrt{19}$. There exist recursion relations to generate $E(n+1, l, m, \tilde{n}, \tilde{l}, \tilde{m}, t, u, v)$, $E(n, l+1, m, \tilde{n}, \tilde{l}, \tilde{m}, t, u, v), \quad E(n, l+1, l+1, \tilde{n}, \tilde{l}, \tilde{m}, t, u, v)$ and $\quad E(n,-l-1,-l-$ $1, \tilde{n}, \tilde{l}, \tilde{m}, t, u, v)$. Recursions are now necessary for $G_{x}^{A}, G_{y}^{A}$ and $G_{z}^{A}$.

\section{Recursion in $l$ and $m$}

With $S(\alpha, \vec{r}-\vec{A}, n, l+1, l+1)=(2 l+1)\left(\left(x-A_{x}\right)+\mathrm{i}\left(y-A_{y}\right)\right) S(\alpha, \vec{r}-\vec{A}, n, l, l)$, we obtain:

$$
S(\alpha, \vec{r}-\vec{A}, n, l+1, l+1) S(\beta, \vec{r}-\vec{B}, \tilde{n}, \tilde{l}, \tilde{m})=
$$




$$
\begin{aligned}
& (2 l+1) \sum_{t, u, v} E(n, l, l, \tilde{n}, \tilde{l}, \tilde{m}, t, u, v)\left(\left(x-A_{x}\right)+\mathrm{i}\left(y-A_{y}\right)\right) \Lambda(\gamma, \vec{r}-\vec{P}, t, u, v)= \\
& \sum_{t, u, v} E(n, l+1, l+1, \tilde{n}, \tilde{l}, \tilde{m}, t, u, v) \Lambda(\gamma, \vec{r}-\vec{P}, t, u, v)
\end{aligned}
$$

In the case of gradients, we obtain:

$$
\begin{aligned}
& \frac{\partial}{\partial A_{x}} S(\alpha, \vec{r}-\vec{A}, n, l+1, l+1) S(\beta, \vec{r}-\vec{B}, \tilde{n}, \tilde{l}, \tilde{m})= \\
& (2 l+1) \frac{\partial}{\partial A_{x}}\left(\sum_{t, u, v} E(n, l, l, \tilde{n}, \tilde{l}, \tilde{m}, t, u, v)\left(\left(x-A_{x}\right)+\mathrm{i}\left(y-A_{y}\right)\right) \Lambda(\gamma, \vec{r}-\vec{P}, t, u, v)\right)= \\
& (2 l+1)\left(\sum_{t, u, v}-E(n, l, l, \tilde{n}, \tilde{l}, \tilde{m}, t, u, v) \Lambda(\gamma, \vec{r}-\vec{P}, t, u, v)+\right. \\
& \left.\left(\left(x-A_{x}\right)+\mathrm{i}\left(y-A_{y}\right)\right) \frac{\partial}{\partial A_{x}}(E(n, l, l, \tilde{n}, \tilde{l}, \tilde{m}, t, u, v) \Lambda(\gamma, \vec{r}-\vec{P}, t, u, v))\right)= \\
& (2 l+1)\left(\sum_{t, u, v}-E(n, l, l, \tilde{n}, \tilde{l}, \tilde{m}, t, u, v) \Lambda(\gamma, \vec{r}-\vec{P}, t, u, v)+\right. \\
& \left.\left(\left(x-A_{x}\right)+\mathrm{i}\left(y-A_{y}\right)\right) G_{x}^{A}(n, l, l, \tilde{n}, \tilde{l}, \tilde{m}, t, u, v) \Lambda(\gamma, \vec{r}-\vec{P}, t, u, v)\right)= \\
& \sum_{t, u, v} G_{x}^{A}(n, l+1, l+1, \tilde{n}, \tilde{l}, \tilde{m}, t, u, v) \Lambda(\gamma, \vec{r}-\vec{P}, t, u, v)
\end{aligned}
$$

By substituting the recursion relation for Hermite polynomials

$$
\left(x-P_{x}\right) \Lambda(\gamma, \vec{r}-\vec{P}, t, u, v)=\frac{1}{2 \gamma} \Lambda(\gamma, \vec{r}-\vec{P}, t+1, u, v)+t \Lambda(\gamma, \vec{r}-\vec{P}, t-1, u, v)
$$

in the penultimate expression of equation 30, we obtain:

$$
\begin{array}{r}
(2 l+1) \sum_{t, u, v}-E(n, l, l, \tilde{n}, \tilde{l}, \tilde{m}, t, u, v) \Lambda(\gamma, \vec{r}-\vec{P}, t, u, v)+ \\
\left(\frac{1}{2 \gamma} \Lambda(\gamma, \vec{r}-\vec{P}, t+1, u, v)+\right. \\
t \Lambda(\gamma, \vec{r}-\vec{P}, t-1, u, v)+ \\
\left(P_{x}-A_{x}\right) \Lambda(\gamma, \vec{r}-\vec{P}, t, u, v)+ \\
\mathrm{i}\left(\frac{1}{2 \gamma} \Lambda(\gamma, \vec{r}-\vec{P}, t, u+1, v)+\right. \\
u \Lambda(\gamma, \vec{r}-\vec{P}, t, u-1, v)+ \\
\left.\left.\left(P_{y}-A_{y}\right) \Lambda(\gamma, \vec{r}-\vec{P}, t, u, v)\right)\right) G_{x}^{A}(n, l, l, \tilde{n}, \tilde{l}, \tilde{m}, t, u, v)
\end{array}
$$

From this, we deduce the following relation: 


$$
\begin{array}{r}
G_{x}^{A}(n, l+1, l+1, \tilde{n}, \tilde{l}, \tilde{m}, t, u, v)= \\
(2 l+1)(-E(n, l, l, \tilde{n}, \tilde{l}, \tilde{m}, t, u, v)+ \\
\quad \frac{1}{2 \gamma} G_{x}^{A}(n, l, l, \tilde{n}, \tilde{l}, \tilde{m}, t-1, u, v)+ \\
(t+1) G_{x}^{A}(n, l, l, \tilde{n}, \tilde{l}, \tilde{m}, t+1, u, v)+ \\
\left(P_{x}-A_{x}\right) G_{x}^{A}(n, l, l, \tilde{n}, \tilde{l}, \tilde{m}, t, u, v)+ \\
\mathrm{i}\left(\frac{1}{2 \gamma} G_{x}^{A}(n, l, l, \tilde{n}, \tilde{l}, \tilde{m}, t, u-1, v)+\right. \\
(u+1) G_{x}^{A}(n, l, l, \tilde{n}, \tilde{l}, \tilde{m}, t, u+1, v)+ \\
\left.\left.\left(P_{y}-A_{y}\right) G_{x}^{A}(n, l, l, \tilde{n}, \tilde{l}, \tilde{m}, t, u, v)\right)\right)
\end{array}
$$

In an analogous way, we obtain the following recursion relation:

$$
\begin{array}{r}
G_{x}^{A}(n, l+1,-l-1, \tilde{n}, \tilde{l}, \tilde{m}, t, u, v)= \\
(2 l+1)(-E(n, l,-l, \tilde{n}, \tilde{l}, \tilde{m}, t, u, v)+ \\
\quad \frac{1}{2 \gamma} G_{x}^{A}(n, l,-l, \tilde{n}, \tilde{l}, \tilde{m}, t-1, u, v)+ \\
(t+1) G_{x}^{A}(n, l,-l, \tilde{n}, \tilde{l}, \tilde{m}, t+1, u, v)+ \\
\left(P_{x}-A_{x}\right) G_{x}^{A}(n, l,-l, \tilde{n}, \tilde{l}, \tilde{m}, t, u, v)- \\
\mathrm{i}\left(\frac{1}{2 \gamma} G_{x}^{A}(n, l,-l, \tilde{n}, \tilde{l}, \tilde{m}, t, u-1, v)+\right. \\
(u+1) G_{x}^{A}(n, l,-l, \tilde{n}, \tilde{l}, \tilde{m}, t, u+1, v)+ \\
\left.\left.\left(P_{y}-A_{y}\right) G_{x}^{A}(n, l,-l, \tilde{n}, \tilde{l}, \tilde{m}, t, u, v)\right)\right)
\end{array}
$$

\section{Recursion in $n$}

With $S(\alpha, \vec{r}-\vec{A}, n+1, l, m)=|\vec{r}-\vec{A}|^{2} S(\alpha, \vec{r}-\vec{A}, n, l, m)$ the following relation can be derived 9 :

$$
\begin{array}{r}
G_{x}^{A}(n+1, l, m, \tilde{n}, \tilde{l}, \tilde{m}, t, u, v)= \\
\frac{1}{4 \gamma^{2}}\left(G_{x}^{A}(n, l, m, \tilde{n}, \tilde{l}, \tilde{m}, t-2, u, v)+\right.
\end{array}
$$




$$
\begin{array}{r}
G_{x}^{A}(n, l, m, \tilde{n}, \tilde{l}, \tilde{m}, t, u-2, v)+ \\
\left.G_{x}^{A}(n, l, m, \tilde{n}, \tilde{l}, \tilde{m}, t, u, v-2)\right)+ \\
\frac{1}{\gamma}\left(\left(P_{x}-A_{x}\right) G_{x}^{A}(n, l, m, \tilde{n}, \tilde{l}, \tilde{m}, t-1, u, v)-\right. \\
E(n, l, m, \tilde{n}, \tilde{l}, \tilde{m}, t-1, u, v)+ \\
\left(P_{y}-A_{y}\right) G_{x}^{A}(n, l, m, \tilde{n}, \tilde{l}, \tilde{m}, t, u-1, v)+ \\
\left.\left(P_{z}-A_{z}\right) G_{x}^{A}(n, l, m, \tilde{n}, \tilde{l}, \tilde{m}, t, u, v-1)\right)+ \\
\left(t+u+v+\frac{3}{2}\right) \\
\left.\gamma\right|^{2}+G_{x}^{A}(n, l, m, \tilde{n}, \tilde{l}, \tilde{m}, t, u, v)- \\
2\left(P_{x}-A_{x}\right) E(n, l, m, \tilde{n}, \tilde{l}, \tilde{m}, t, u, v)+ \\
\left(P_{x}-A_{x}\right)(t+1) G_{x}^{A}(n, l, m, \tilde{n}, \tilde{l}, \tilde{m}, t+1, u, v)- \\
(t+1) E(n, l, m, \tilde{n}, \tilde{l}, \tilde{m}, t+1, u, v)+ \\
\left(P_{y}-A_{y}\right)(u+1) G_{x}^{A}(n, l, m, \tilde{n}, \tilde{l}, \tilde{m}, t, u+1, v)+ \\
\left.\left(P_{z}-A_{z}\right)(v+1) G_{x}^{A}(n, l, m, \tilde{n}, \tilde{l}, \tilde{m}, t, u, v+1)\right)+ \\
(t+2)(t+1) G_{x}^{A}(n, l, m, \tilde{n}, \tilde{l}, \tilde{m}, t+2, u, v)+ \\
(u+2)(u+1) G_{x}^{A}(n, l, m, \tilde{n}, \tilde{l}, \tilde{m}, t, u+2, v)+ \\
(v+1) G_{x}^{A}(n, l, m, \tilde{n}, \tilde{l}, \tilde{m}, t, u, v+2)
\end{array}
$$

\section{Recursion in $l$}

Using that $S(\alpha, \vec{r}-\vec{A}, n, l+1, m)=\left((2 l+1)\left(z-A_{z}\right) S(\alpha, \vec{r}-\vec{A}, n, l, m)-|\vec{r}-\vec{A}|^{2}(l+\right.$ $|m|) S(\alpha, \vec{r}-\vec{A}, n, l-1, m)) /(l-|m|+1)$ the following relation can be derived $\mathbb{1}$ :

$$
\begin{array}{r}
G_{x}^{A}(n, l+1, m, \tilde{n}, \tilde{l}, \tilde{m}, t, u, v)= \\
\left(( 2 l + 1 ) \left(\frac{G_{x}^{A}(n, l, m, \tilde{n}, \tilde{l}, \tilde{m}, t, u, v-1)}{2 \gamma}+\right.\right. \\
\left(P_{z}-A_{z}\right) G_{x}^{A}(n, l, m, \tilde{n}, \tilde{l}, \tilde{m}, t, u, v)+ \\
\left.(v+1) G_{x}^{A}(n, l, m, \tilde{n}, \tilde{l}, \tilde{m}, t, u, v+1)\right)- \\
(l+|m|)\left(\left(G_{x}^{A}(n, l-1, m, \tilde{n}, \tilde{l}, \tilde{m}, t-2, u, v)+\right.\right.
\end{array}
$$




$$
\begin{array}{r}
G_{x}^{A}(n, l-1, m, \tilde{n}, \tilde{l}, \tilde{m}, t, u-2, v)+ \\
\left.G_{x}^{A}(n, l-1, m, \tilde{n}, \tilde{l}, \tilde{m}, t, u, v-2)\right) /(2 \gamma)^{2}+ \\
\left(\left(P_{x}-A_{x}\right) G_{x}^{A}(n, l-1, m, \tilde{n}, \tilde{l}, \tilde{m}, t-1, u, v)-\right. \\
E(n, l-1, m, \tilde{n}, \tilde{l}, \tilde{m}, t-1, u, v)+ \\
\left(P_{y}-A_{y}\right) G_{x}^{A}(n, l-1, m, \tilde{n}, \tilde{l}, \tilde{m}, t, u-1, v)+ \\
\left.\left(P_{z}-A_{z}\right) G_{x}^{A}(n, l-1, m, \tilde{n}, \tilde{l}, \tilde{m}, t, u, v-1)\right) / \gamma+ \\
\left(t+u+v+\frac{3}{2}\right) \\
\gamma\left(\vec{P}^{2}+G_{x}^{A}(n, l-1, m, \tilde{n}, \tilde{l}, \tilde{m}, t, u, v)-\right. \\
2\left(P_{x}-A_{x}\right) E(n, l-1, m, \tilde{n}, \tilde{l}, \tilde{m}, t, u, v)+ \\
2\left(P_{x}-A_{x}\right)(t+1) G_{x}^{A}(n, l-1, m, \tilde{n}, \tilde{l}, \tilde{m}, t+1, u, v)- \\
(t+1) E(n, l-1, m, \tilde{n}, \tilde{l}, \tilde{m}, t+1, u, v)+ \\
\left(P_{y}-A_{y}\right)(u+1) G_{x}^{A}(n, l-1, m, \tilde{n}, \tilde{l}, \tilde{m}, t, u+1, v)+ \\
\left.\left(P_{z}-A_{z}\right)(v+1) G_{x}^{A}(n, l-1, m, \tilde{n}, \tilde{l}, \tilde{m}, t, u, v+1)\right)+ \\
(t+2)(t+1) G_{x}^{A}(n, l-1, m, \tilde{n}, \tilde{l}, \tilde{m}, t+2, u, v)+ \\
\left.\left.(v+1) G_{x}^{A}(n, l-1, m, \tilde{n}, \tilde{l}, \tilde{m}, t, u, v+2)\right)\right) /(l-|m|+1) \\
(u+1) G_{x}^{A}(n, l-1, m, \tilde{n}, \tilde{m}, t, u+2, v)+
\end{array}
$$

The recursion relations for the $G_{x}^{A}$-coefficients are similar to those for the $E$-coefficients2 2 , with the extra terms arising from the derivative $\frac{\partial}{\partial A_{x}}\left(x-A_{x}\right)$ for the recursion in $l$ and $m$, and from the derivative $\frac{\partial}{\partial A_{x}}|\vec{r}-\vec{A}|^{2}$ for the recursion in $l$ and the recursion in $n$.

This can similarly be done for $G_{y}^{A}$ and $G_{z}^{A}$. Finally, all $G$-coefficients are zero for the case $t+u+v>2 n+2 \tilde{n}+l+\tilde{l}+1$ and for all negative values of $t, u$ or $v$.

\section{B. Gradients with respect to other centers}

To obtain the derivatives with respect to center $B$, the following relation is used 31 (note $\vec{R}=\vec{A}-\vec{B}):$

$$
\frac{\partial}{\partial A_{x}}=\frac{\partial}{\partial P_{x}} \frac{\partial P_{x}}{\partial A_{x}}+\frac{\partial}{\partial R_{x}} \frac{\partial R_{x}}{\partial A_{x}}=
$$




$$
\frac{\alpha}{\gamma} \frac{\partial}{\partial P_{x}}+\frac{\partial}{\partial R_{x}}
$$

and

$$
\begin{array}{r}
\frac{\partial}{\partial B_{x}}=\frac{\partial}{\partial P_{x}} \frac{\partial P_{x}}{\partial B_{x}}+\frac{\partial}{\partial R_{x}} \frac{\partial R_{x}}{\partial B_{x}}= \\
\frac{\beta}{\gamma} \frac{\partial}{\partial P_{x}}-\frac{\partial}{\partial R_{x}}
\end{array}
$$

Therefore,

$$
\frac{\partial}{\partial A_{x}}+\frac{\partial}{\partial B_{x}}=\frac{\partial}{\partial P_{x}}
$$

This means that

$$
\begin{array}{r}
\frac{\partial}{\partial B_{x}} \sum_{t, u, v} E(n, l, m, \tilde{n}, \tilde{l}, \tilde{m}, t, u, v) \Lambda(\gamma, \vec{r}-\vec{P}, t, u, v)= \\
\left(\frac{\partial}{\partial P_{x}}-\frac{\partial}{\partial A_{x}}\right) \sum_{t, u, v} E(n, l, m, \tilde{n}, \tilde{l}, \tilde{m}, t, u, v) \Lambda(\gamma, \vec{r}-\vec{P}, t, u, v)
\end{array}
$$

Applying equation 25, we obtain:

$$
\begin{aligned}
& \sum_{t, u, v} G_{x}^{B}(n, l, m, \tilde{n}, \tilde{l}, \tilde{m}, t, u, v) \Lambda(\gamma, \vec{r}-\vec{P}, t, u, v)= \\
& \sum_{t, u, v} E(n, l, m, \tilde{n}, \tilde{l}, \tilde{m}, t, u, v) \Lambda(\gamma, \vec{r}-\vec{P}, t+1, u, v) \\
& -\sum_{t, u, v} G_{x}^{A}(n, l, m, \tilde{n}, \tilde{l}, \tilde{m}, t, u, v) \Lambda(\gamma, \vec{r}-\vec{P}, t, u, v)
\end{aligned}
$$

Finally, we conclude:

$$
G_{x}^{B}(n, l, m, \tilde{n}, \tilde{l}, \tilde{m}, t, u, v)=E(n, l, m, \tilde{n}, \tilde{l}, \tilde{m}, t-1, u, v)-G_{x}^{A}(n, l, m, \tilde{n}, \tilde{l}, \tilde{m}, t, u, v)
$$

and similar for derivatives with respect to $y$ and $z$ direction. All the integrals can be expressed with the help of the E-coefficients. Taking the derivative therefore reduces to replacing $E$ with the corresponding $G_{x}^{A}, G_{y}^{A}$, etc coefficients. 
As an example, we show how overlap integral and gradient are obtained. First, the overlap is computed as follows:

$$
\begin{aligned}
& \int S(\alpha, \vec{r}-\vec{A}, n, l, m) S(\beta, \vec{r}-\vec{B}, \tilde{n}, \tilde{l}, \tilde{m}) \mathrm{d}^{3} \mathrm{r}= \\
& \int \sum_{t, u, v} E(n, l, m, \tilde{n}, \tilde{l}, \tilde{m}, t, u, v) \Lambda(\gamma, \vec{r}-\vec{P}, t, u, v) \mathrm{d}^{3} \mathrm{r}= \\
& E(n, l, m, \tilde{n}, \tilde{l}, \tilde{m}, 0,0,0)\left(\frac{\pi}{\gamma}\right)^{\frac{3}{2}}
\end{aligned}
$$

We have used that

$$
\int \Lambda(\gamma, \vec{r}, t, u, v) \mathrm{d}^{3} \mathrm{r}=\left(\frac{\pi}{\gamma}\right)^{\frac{3}{2}} \delta_{t 0} \delta_{u 0} \delta_{v 0}
$$

because of the orthogonality of the Hermite Gaussian type functions $\left(\delta_{t 0}\right.$ is the Kronecker delta).

The gradient is computed similarly:

$$
\begin{aligned}
& \frac{\partial}{\partial A_{x}} \int S(\alpha, \vec{r}-\vec{A}, n, l, m) S(\beta, \vec{r}-\vec{B}, \tilde{n}, \tilde{l}, \tilde{m}) \mathrm{d}^{3} \mathrm{r}= \\
& \int \sum_{t, u, v} G_{x}^{A}(n, l, m, \tilde{n}, \tilde{l}, \tilde{m}, t, u, v) \Lambda(\gamma, \vec{r}-\vec{P}, t, u, v) \mathrm{d}^{3} \mathrm{r}= \\
& G_{x}^{A}(n, l, m, \tilde{n}, \tilde{l}, \tilde{m}, 0,0,0)\left(\frac{\pi}{\gamma}\right)^{\frac{3}{2}}
\end{aligned}
$$

In our implementation, we therefore compute the gradient of the two Gaussians which are associated with the integrals, by replacing $E$-coefficients with $G$-coefficients. As a consequence, if an operator, which might appear in the integral, has a nonvanishing derivative (such as, for example, the nuclear attraction), this must be taken into account additionally. This derivative with respect to the third center can be obtained by applying translational invariance with respect to a simultaneous uniform translation of the three centers. In the case of bielectronic integrals, products with two E-coefficients appear. Obviously, when differentiating, the corresponding rules of differentiating a product must be applied and two derivative terms appear, each of them consisting of a product of one set of $E-$ and one set of $G$-coefficients. Finally, the nuclear-nuclear term must be differentiated which is trivial. 
It is interesting to compare this implementation with that of the Namur group 14 where also gradients within the McMurchie-Davidson algorithm are computed. Whereas our scheme computes the derivatives of the two Gaussians appearing in the integral and a possibly necessary derivative of an operator is obtained by applying translational invariance, the alternative implementation 14 relies on explicitly computing derivatives of $E$-coefficients and of the auxiliary function $27 R_{t, u, v}$ appearing in the integrals.

\section{TOTAL ENERGY}

The total energy consists of kinetic energy, Coulomb energy (nuclear-nuclear repulsion, nuclear-electron attraction and electron-electron repulsion), and exchange energy. We assume that all the orbitals are either empty or doubly occupied.

\section{A. Kinetic energy}

The kinetic energy of the electrons is obtained as:

$$
\begin{array}{r}
E^{\text {kinetic }}=\sum_{\vec{g}, \mu, \nu} P_{\nu \vec{g} \mu \overrightarrow{0}} \int \phi_{\mu}\left(\vec{r}-\vec{A}_{\mu}\right)\left(-\frac{1}{2} \Delta_{\vec{r}}\right) \phi_{\nu}\left(\vec{r}-\vec{A}_{\nu}-\vec{g}\right) \mathrm{d}^{3} \mathrm{r}= \\
\sum_{\vec{g}, \mu, \nu} P_{\nu \vec{g} \mu \overrightarrow{0}} T_{\mu \overrightarrow{0} \nu \vec{g}}
\end{array}
$$

\section{B. Exchange energy}

The exchange energy is obtained as:

$$
\begin{aligned}
& E^{\mathrm{exch}-\mathrm{el}}= \\
& -\frac{1}{4} \sum_{\vec{g}, \mu, \nu} P_{\nu \vec{g} \mu \overrightarrow{0}} \sum_{\vec{h}, \vec{n}, \tau, \sigma} P_{\sigma \vec{h}+\vec{n} \tau \vec{n}} \\
& \int \frac{\phi_{\mu}\left(\vec{r}-\overrightarrow{A_{\mu}}\right) \phi_{\tau}\left(\vec{r}-\vec{A}_{\tau}-\vec{n}\right) \phi_{\nu}\left(\vec{r}^{\prime}-\vec{A}_{\nu}-\vec{g}\right) \phi_{\sigma}\left(\vec{r}^{\prime}-\vec{A}_{\sigma}-\vec{h}-\vec{n}\right)}{\left|\vec{r}-\vec{r}^{\prime}\right|} \mathrm{d}^{3} \mathrm{r} \mathrm{d}^{3} \mathrm{r}^{\prime}= \\
& -\frac{1}{4} \sum_{\vec{g}, \mu, \nu} P_{\nu \vec{g} \mu \overrightarrow{0}} \sum_{\vec{h}, \tau, \sigma} P_{\sigma \vec{h} \tau \overrightarrow{0}} X_{\mu \overrightarrow{0} \nu \vec{g} \tau \overrightarrow{0} \sigma \vec{h}}
\end{aligned}
$$


where we have exploited translational invariance of the density matrix with respect to direct lattice vectors $\vec{n}: P_{\sigma \vec{h}+\vec{n} \tau \vec{n}}=P_{\sigma \vec{h} \overrightarrow{0}}$.

We can define a Fock operator for the exchange energy which is

$$
F_{\mu \overrightarrow{0} \nu \vec{g}}^{\operatorname{exch}-\mathrm{el}}=-\frac{1}{2} \sum_{\vec{h}, \tau, \sigma} P_{\sigma \vec{h} \tau \overrightarrow{0}} X_{\mu \overrightarrow{0} \nu \vec{g} \tau \overrightarrow{0} \sigma \vec{h}}
$$

\section{Coulomb energy}

Both kinetic energy and exchange energy must converge independently. However, a separation of the contributions to the Coulomb energy is not possible: for example, in a one dimensional periodic system with lattice constant $a$, and $n$ being an index enumerating the cells, the electron-electron interaction per unit cell would have contributions like:

$$
\sum_{n=1}^{\infty} \frac{1}{n a}
$$

This sum is divergent (similarly in two and three dimensions). Therefore, in CRYSTAL

a scheme based on the Ewald method is used to sum the interactions 22.26 . We only quote the results for the individual contributions:

1. Nuclear-nuclear repulsion:

$$
E^{\mathrm{NN}}=\frac{1}{2} \sum_{a, b} Z_{a} Z_{b} A\left(\vec{A}_{b}-\vec{A}_{a}\right)
$$

\section{Nuclear-electron attraction:}

The energy $\frac{1}{2} E_{\mathrm{NE}}$, which is the Ewald energy of the nuclei in the primitive unit cell with the all the electrons of all cells, is the same as the energy $\frac{1}{2} E_{\mathrm{EN}}$, which is the Ewald energy of the electrons of the primitive unit cell with all the nuclei in all cells, as long as no approximations are introduced 30 . CRYSTAL uses the following expression as the sum of these interactions: 


$$
E^{\mathrm{coul}-\mathrm{nuc}}=\sum_{\vec{g}, \mu, \nu} P_{\nu \vec{g} \mu \overrightarrow{0}} F_{\mu \overrightarrow{0} \nu \vec{g}}^{\mathrm{coul}-\mathrm{nuc}}
$$

with the Fock matrix $F_{\mu \overrightarrow{0} \nu \vec{g}}^{\mathrm{coul}-\text { nuc }}$ containing the nuclear-electron contributions defined as

$$
F_{\mu \overrightarrow{0} \nu \vec{g}}^{\mathrm{coul}-\mathrm{nuc}}=-\sum_{a} Z_{a} \int \phi_{\mu}\left(\vec{r}-\vec{A}_{\mu}\right) \phi_{\nu}\left(\vec{r}-A_{\nu}-\vec{g}\right) A\left(\vec{r}-\vec{A}_{a}\right) \mathrm{d}^{3} \mathrm{r}
$$

\section{Electron-electron repulsion:}

$$
E^{\mathrm{coul}-\mathrm{el}}=\frac{1}{2} \sum_{\vec{g}, \mu, \nu} P_{\nu \vec{g} \mu \overrightarrow{0}} F_{\mu \overrightarrow{0} \nu \vec{g}}^{\mathrm{coul}-\mathrm{el}}
$$

with the Fock matrix $F_{\mu \overrightarrow{0} \nu \vec{g}}^{\mathrm{coul}-\mathrm{el}}$ containing the electron-electron contributions defined as

$$
F_{\mu \overrightarrow{0} \nu \vec{g}}^{\mathrm{coul}-\mathrm{el}}=-Q S_{\mu \overrightarrow{0} \nu \vec{g}}+\sum_{\vec{h}, \tau, \sigma} P_{\sigma \vec{h} \tau \overrightarrow{0}} C_{\mu \overrightarrow{0} \nu \vec{g} \tau \overrightarrow{0} \sigma \vec{h}}-\sum_{c} \sum_{l=0}^{L} \sum_{m=-l}^{l} \eta_{l}^{m}\left(\rho_{c} ; \vec{A}_{c}\right) M_{l \mu \overrightarrow{0} \nu \vec{g} c}^{m}
$$

\section{Total energy}

Finally, the total energy can be expressed as

$$
\begin{aligned}
& E^{\text {total }}=E^{\text {kinetic }}+E^{\mathrm{NN}}+E^{\text {coul-nuc }}+E^{\text {coul-el }}+E^{\text {exch-el }}= \\
& =E^{\mathrm{NN}}+\sum_{\vec{g}, \mu, \nu} P_{\nu \vec{g} \mu \overrightarrow{0}} T_{\mu \overrightarrow{0} \nu \vec{g}} \\
& -\sum_{\vec{g}, \mu, \nu} P_{\nu \vec{g} \mu \overrightarrow{0}} \sum_{a} Z_{a} \int \phi_{\mu}\left(\vec{r}-\overrightarrow{A_{\mu}}\right) \phi_{\nu}\left(\vec{r}-\vec{A}_{\nu}-\vec{g}\right) A\left(\vec{r}-\vec{A}_{a}\right) \mathrm{d}^{3} \mathrm{r} \\
& +\frac{1}{2} \sum_{\vec{g}, \mu, \nu} P_{\nu \vec{g} \mu \overrightarrow{0}}\left(-Q S_{\mu \overrightarrow{0} \nu \vec{g}}+\sum_{\vec{h}, \tau, \sigma} P_{\sigma \vec{h} \tau \overrightarrow{0}} C_{\mu \overrightarrow{0} \nu \vec{g} \tau \overrightarrow{0} \sigma \vec{h}}-\sum_{c} \sum_{l=0}^{L} \sum_{m=-l}^{l} \eta_{l}^{m}\left(\rho_{c} ; \vec{A}_{c}\right) M_{l \mu \overrightarrow{0} \nu \vec{g} c}^{m}\right) \\
& -\frac{1}{4} \sum_{\vec{g}, \mu, \nu} P_{\nu \vec{g} \mu \overrightarrow{0}} \sum_{\vec{h}, \tau, \sigma} P_{\sigma \vec{h} \tau \overrightarrow{0}} X_{\mu \overrightarrow{0} \nu \vec{g} \tau \overrightarrow{0} \sigma \vec{h}}
\end{aligned}
$$

The Fock operator used has the structure:

$$
F_{\mu \overrightarrow{0} \nu \vec{g}}^{\text {total }}=T_{\mu \overrightarrow{0} \nu \vec{g}}+F_{\mu \overrightarrow{0} \nu \vec{g}}^{\mathrm{coul}-\mathrm{nuc}}+F_{\mu \overrightarrow{0} \nu \vec{g}}^{\mathrm{coul}-\mathrm{el}}+F_{\mu \overrightarrow{0} \nu \vec{g}}^{\mathrm{exch}-\mathrm{el}}
$$

We note that this expression for the Fock operator would be exact if we could guarantee that the penetration depth and screening was symmetric. This would require that 
$C_{\mu \overrightarrow{0} \nu \vec{g} \tau \overrightarrow{0} \sigma \vec{h}}=C_{\tau \overrightarrow{0} \sigma \vec{h} \mu \overrightarrow{0} \nu \vec{g}}$ should always hold. This, however, as aforementioned, cannot be guaranteed because the truncation is not necessarily symmetric. In addition, the screening of the exchange interaction is not necessarily symmetric. Therefore, an inaccuracy in the Fock operator will show up which will be stronger the more asymmetric the truncation in the energy expression is.

The total energy can be expressed as

$$
E^{\text {total }}=E^{\mathrm{NN}}+\sum_{\vec{g}, \mu, \nu} P_{\nu \vec{g} \mu \overrightarrow{0}}\left(T_{\mu \overrightarrow{0} \nu \vec{g}}+F_{\mu \overrightarrow{0} \nu \vec{g}}^{\mathrm{coul}-\mathrm{nuc}}+\frac{1}{2}\left(F_{\mu \overrightarrow{0} \nu \vec{g}}^{\mathrm{coul}-\mathrm{el}}+F_{\mu \overrightarrow{0} \nu \vec{g}}^{\mathrm{exch}-\mathrm{el}}\right)\right)
$$

and the Hartree-Fock equations become as in equation 5. In ref. 26, it was pointed out that the quantity $Q S_{\mu \overrightarrow{0} \nu \vec{g}}$ can be removed from the Fock operator which has been done in CRYSTAL. This leads to eigenvalues shifted by $Q$ as we now use the modified equation

$$
\sum_{\nu}\left(F_{\mu \nu}^{\mathrm{total}}(\vec{k})+Q S_{\mu \nu}(\vec{k})\right) a_{\nu i}(\vec{k})=\sum_{\nu} S_{\mu \nu}(\vec{k}) a_{\nu i}(\vec{k})\left(\epsilon_{i}(\vec{k})+Q\right)
$$

\section{GRADIENT OF THE TOTAL ENERGY}

The force on the nuclei can be calculated similarly to the molecular case日包. The derivatives of all the integrals are necessary, and the derivative of the density matrix is expressed with the help of the energy-weighted density matrix. One important assumption is that

$$
B_{\mu \overrightarrow{0} \nu \vec{g} \tau \vec{n} \sigma \vec{n}+\vec{h}}=B_{\tau \vec{n} \sigma \vec{n}+\vec{h} \mu \overrightarrow{0} \nu \vec{g}}=B_{\sigma \vec{n}+\vec{h} \tau \vec{n} \nu \vec{g} \mu \overrightarrow{0}}
$$

holds. Taking the derivative leads, for example, to terms like the following

$$
\begin{aligned}
& \frac{\partial}{\partial \overrightarrow{A_{i}}}\left(\sum_{\vec{g}, \mu, \nu} P_{\nu \vec{g} \mu \overrightarrow{0}} \sum_{\vec{h}, \tau, \sigma} P_{\sigma \vec{h} \tau \overrightarrow{0}} C_{\mu \overrightarrow{0} \nu \vec{g} \tau \overrightarrow{0} \sigma \vec{h}}\right)= \\
& \sum_{\vec{g}, \mu, \nu}\left(\frac{\partial}{\partial \vec{A}_{i}} P_{\nu \vec{g} \mu \overrightarrow{0}}\right) \sum_{\vec{h}, \tau, \sigma} P_{\sigma \vec{h} \tau \overrightarrow{0}} C_{\mu \overrightarrow{0} \nu \vec{g} \tau \overrightarrow{0} \sigma \vec{h}}+\sum_{\vec{g}, \mu, \nu} P_{\nu \vec{g} \mu \overrightarrow{0}} \sum_{\vec{h}, \tau, \sigma}\left(\frac{\partial}{\partial \overrightarrow{A_{i}}} P_{\sigma \vec{h} \tau \overrightarrow{0}}\right) C_{\mu \overrightarrow{0} \nu \vec{g} \tau \overrightarrow{0} \sigma \vec{h}} \\
& +\sum_{\vec{g}, \mu, \nu} P_{\nu \vec{g} \mu \overrightarrow{0}} \sum_{\vec{h}, \tau, \sigma} P_{\sigma \vec{h} \tau \overrightarrow{0}}\left(\frac{\partial}{\partial \overrightarrow{A_{i}}} C_{\mu \overrightarrow{0} \nu \vec{g} \tau \overrightarrow{0} \sigma \vec{h}}\right)
\end{aligned}
$$

When equation 59 holds, we rename the indices in the second addend and obtain: 


$$
2 \sum_{\vec{g}, \mu, \nu}\left(\frac{\partial}{\partial \overrightarrow{A_{i}}} P_{\nu \vec{g} \mu \overrightarrow{0}}\right) \sum_{\vec{h}, \tau, \sigma} P_{\sigma \vec{h} \tau \overrightarrow{0}} C_{\mu \overrightarrow{0} \nu \vec{g} \tau \overrightarrow{0} \sigma \vec{h}}+\sum_{\vec{g}, \mu, \nu} P_{\nu \vec{g} \mu \overrightarrow{0}} \sum_{\vec{h}, \tau, \sigma} P_{\sigma \vec{h} \tau \overrightarrow{0}}\left(\frac{\partial}{\partial \overrightarrow{A_{i}}} C_{\mu \overrightarrow{0} \nu \vec{g} \tau \overrightarrow{0} \sigma \vec{h}}\right)
$$

We derived the equation for the force this way although equation 59 does not always hold. Therefore, inaccuracies will appear when equation 59 is strongly violated. The full force is obtained as:

$$
\begin{aligned}
& \vec{F}_{A_{i}}=-\frac{\partial E^{\text {total }}}{\partial \vec{A}_{i}}= \\
& -\sum_{\vec{g}, \mu, \nu} P_{\nu \vec{g} \mu \overrightarrow{0}} \frac{\partial T_{\mu \overrightarrow{0} \nu \vec{g}}}{\partial \vec{A}_{i}}-\frac{\partial E^{\mathrm{NN}}}{\partial \vec{A}_{i}} \\
& +\sum_{\vec{g}, \mu, \nu} P_{\nu \vec{g} \mu \overrightarrow{0}} \sum_{a} Z_{a} \frac{\partial}{\partial \overrightarrow{A_{i}}}\left[\int \phi_{\mu}\left(\vec{r}-\overrightarrow{A_{\mu}}\right) \phi_{\nu}\left(\vec{r}-\overrightarrow{A_{\nu}}-\vec{g}\right) A\left(\vec{r}-\vec{A}_{a}\right) \mathrm{d}^{3} \mathrm{r}\right] \\
& -\frac{1}{2} \sum_{\vec{g}, \mu, \nu} P_{\nu \vec{g} \mu \overrightarrow{0}}\left\{-S_{\mu \overrightarrow{0} \nu \vec{g}} \frac{2 \pi}{3 V} \sum_{c} \sum_{\vec{h}, \sigma, \tau \in c} P_{\sigma \vec{h} \tau \overrightarrow{0}}\right. \\
& \frac{\partial}{\partial \overrightarrow{A_{i}}} \int\left[-\phi_{\tau}\left(\vec{r}-\vec{A}_{\tau}\right) \phi_{\sigma}\left(\vec{r}-\vec{A}_{\sigma}-\vec{h}\right)\right. \\
& \left.+\sum_{l=0}^{L} \sum_{m=-l}^{l} \int \phi_{\tau}\left(\vec{r}^{\prime}-\vec{A}_{\tau}\right) \phi_{\sigma}\left(\vec{r}^{\prime}-\overrightarrow{A_{\sigma}}-\vec{h}\right) X_{l}^{m}\left(\vec{r}^{\prime}-\vec{A}_{c}\right) \mathrm{d}^{3} \mathrm{r}^{\prime} \delta_{l}^{m}\left(\overrightarrow{A_{c}}, \vec{r}\right)\right] r^{2} \mathrm{~d}^{3} \mathrm{r} \\
& +\sum_{\tau, \sigma} P_{\sigma \vec{h} \tau \overrightarrow{0}} \frac{\partial C_{\mu \overrightarrow{0} \nu \vec{g} \tau \overrightarrow{0} \sigma \vec{h}}}{\partial \vec{A}_{i}} \\
& \left.-\sum_{c} \sum_{l=0}^{L} \sum_{m=-l}^{l} \sum_{\vec{h}, \tau \in c, \sigma} P_{\sigma \vec{h} \tau \overrightarrow{0}} \frac{\partial}{\partial \overrightarrow{A_{i}}}\left[\int \phi_{\tau}\left(\vec{r}-\vec{A}_{\tau}\right) \phi_{\sigma}\left(\vec{r}-\vec{A}_{\sigma}-\vec{h}\right) X_{l}^{m}\left(\vec{r}-\overrightarrow{A_{c}}\right) \mathrm{d}^{3} \mathrm{r} M_{l \mu \overrightarrow{0} \nu \vec{g} c}^{m}\right]\right\} \\
& +\frac{1}{4} \sum_{\vec{g}, \mu, \nu} P_{\nu \vec{g} \mu \overrightarrow{0}} \sum_{\vec{h}, \tau, \sigma} P_{\sigma \vec{h} \tau \overrightarrow{0}} \frac{\partial X_{\mu \overrightarrow{0} \nu \vec{g} \tau \overrightarrow{0} \sigma \vec{h}}}{\partial \overrightarrow{A_{i}}} \\
& -\sum_{\vec{g}, \mu, \nu} \frac{\partial S_{\mu \overrightarrow{0} \nu \vec{g}}}{\partial \vec{A}_{i}} \int_{B Z} \exp (\mathrm{i} \vec{k} \vec{g}) \sum_{j} 2 a_{\nu j}(\vec{k}) a_{\mu j}^{*}(\vec{k})\left(\epsilon_{j}(\vec{k})+Q\right) \Theta\left(\epsilon_{F}-\epsilon_{j}(\vec{k})-Q\right) \mathrm{d}^{3} \mathrm{k}
\end{aligned}
$$

The last addend is the energy weighted density matrix; the integral is over the first Brillouin zone. It is worthwhile mentioning that the factor $P_{\nu \vec{g} \mu \overrightarrow{0}} S_{\mu \overrightarrow{0} \nu \vec{g}}$ is equal to the number of electrons in the unit cell and therefore its derivative with respect to $\vec{A}_{i}$ vanishes. We note three important points:

- Equation 62 is correct for the exact solution of the Hartree-Fock equations. Thus, in practice, a well converged solution is necessary to achieve accurate forces. 
- The energy-weighted density matrix is $\vec{k}$-dependent. Therefore, the accuracy of the forces will become dependent on the number of $\vec{k}$-points.

- The derivation of equation 62 assumes that equation 59 holds. The treatment of the Coulomb series with finite penetration depth leads to an asymmetry associated with ITOL2 in the CRYSTAL code. In addition, in the treatment of the exchange series an asymmetry can be introduced if the screening parameters (ITOL4 and ITOL5 in the CRYSTAL code) are chosen differently. Therefore, the choice of ITOL2, ITOL4 and ITOL5 will influence the accuracy of the gradients.

\section{RESULTS FROM TEST CALCULATIONS}

With a few examples, we want to illustrate the accuracy of the analytically computed gradients.

In table [I, all the integrals are evaluated without approximation and the analytical derivative agrees to five digits with the numerical derivative. As the numerical derivative is only accurate up to five digits, this is certainly satisfying.

In table $\mathbb{1}$ the variation in accuracy when penetration depth and overlap criteria are altered, is displayed. As described in the article, lowering ITOL2 to low values leads to inaccuracies in the gradients. Lowering only one of the parameters ITOL4 or ITOL5 also leads to inaccuracies, whereas lowering both to the same value gives an analytical gradient which is consistent with the numerical gradient - however, as a value of 1 for ITOL4 and ITOL5 was chosen, energy and force are completely different from calculations with reasonable values for ITOL4 and ITOL5. The parameter ITOL1, which selects the oneelectron and Coulomb integrals according to the overlap, can lead to numerical instabilities in the energy calculation and inaccurate gradients when chosen too low, and should therefore be reasonably high. The parameter ITOL3 does not influence the accuracy of the gradients: although when chosen much too low with a value of e.g. 1, numerical and analytical gradient still agree. The default values for the $I T O L$-parameters for the energy calculation are 6, 6, 
6, 6 and 12. In all calculations performed so far, these default values did not lead to serious errors for the gradients.

Another example (table III) is the CO molecule arranged as a single molecule ("molecule"), as a molecule periodically repeated in one dimension ("polymer"), in two dimensions ("slab") and in three dimensions ("bulk"). The forces agree well, and it is demonstrated that using stricter real-space truncation parameters improves the agreement. The forces seem to be relatively insensitive to the number of sampling points and changing their number changed the error in the forces only slightly.

Finally, in table $[\mathrm{V}$ we compare analytical and numerical derivatives for $\mathrm{MgO}$ when moving the oxygen atom in x-direction which would correspond to a longitudinal phonon. Again, agreement is to the order of $10^{-5} \frac{E_{h}}{a_{B}}$ (default ITOL parameters were used).

\section{CONCLUSION}

We presented the theory of analytic Hartree-Fock gradients for periodic systems. This has been implemented in the code CRYSTAL which is to the best of our knowledge the first implementation of Hartree-Fock gradients in systems periodic in 2 and 3 dimensions. The results are in excellent agreement with numerical derivatives.

Future directions will be the improvement of the efficiency of the code (implementation of symmetry and various technical improvements), derivatives with respect to the lattice vector, as well as an extension to metallic systems.

\section{ACKNOWLEDGMENTS}

The authors would like to acknowledge support from EPSRC grant GR/K90661. 


\section{REFERENCES}

${ }^{1}$ P. Pulay, Mol. Phys. 17, 197 (1969).

${ }^{2}$ S. Bratoŭ, in Calcul des fonctions d'onde moléculaire, Colloq. Int. C. N. R. S. 82, 287 (1958).

${ }^{3}$ P. Pulay, Adv. Chem. Phys. 69, 241 (1987).

${ }^{4}$ P. Pulay, in Applications of Electronic Structure Theory, edited by H. F. Schaefer III, 153 (Plenum, New York, 1977).

${ }^{5}$ H. B. Schlegel, Adv. Chem. Phys. 67, 249 (1987).

${ }^{6}$ T. Helgaker and P. Jørgensen, Adv. in Quantum Chem. 19, 183 (1988)

${ }^{7}$ H. Teramae, T. Yamabe, C. Satoko and A. Imamura, Chem. Phys. Lett. 101, 149 (1983);

H. Teramae, T. Yamabe and A. Imamura, J. Chem. Phys. 81, 3564 (1984).

${ }^{8}$ M. Kertesz, Chem. Phys. Lett. 106, 443 (1984).

${ }^{9}$ S. Suhai, Phys. Rev. B 27, 3506 (1983); S. Suhai, Chem. Phys. Lett. 96, 619 (1983).

10 J.-Q. Sun and R. J. Bartlett, J. Chem. Phys. 104, 8553 (1996).

${ }^{11}$ S. Hirata and S. Iwata, J. Chem. Phys. 109, 4147 (1998).

12 S. Hirara and S. Iwata, J. Chem. Phys. 107, 10075 (1997); S. Hirata, H. Torii and M. Tasumi, Phys. Rev. B 57, 11994 (1998); S. Hirata and S. Iwata, J. Chem. Phys. 108, 7901 (1998); S. Hirata and S. Iwata, J. Phys. Chem. A 102, 8426 (1998).

${ }^{13}$ S. Hirata and S. Iwata, J. Mol. Struct.: THEOCHEM 451, 121 (1998).

${ }^{14}$ D. Jacquemin, J.-M. André and B. Champagne, J. Chem. Phys. 111, 5306 (1999); J. Chem. Phys. 111, 5324 (1999).

${ }^{15}$ K. N. Kudin and G. E. Scuseria, Phys. Rev. B 61, 16440 (2000). 
${ }^{16}$ V. R. Saunders, R. Dovesi, C. Roetti, M. Causà, N. M. Harrison, R. Orlando, C. M. Zicovich-Wilson, CRYstal 98 User's Manual, Theoretical Chemistry Group, University of Torino (1998).

${ }^{17}$ C. Pisani and R. Dovesi, Int. J. Quantum Chem. 17, 501 (1980); R. Dovesi and C. Roetti, Int. J. Quantum Chem. 17, 517 (1980).

${ }^{18}$ C. Pisani, R. Dovesi, and C. Roetti, Hartree-Fock Ab Initio Treatment of Crystalline Systems, edited by G. Berthier et al, Lecture Notes in Chemistry Vol. 48 (Springer, Berlin, 1988).

${ }^{19}$ V. R. Saunders, N. M. Harrison, R. Dovesi, C. Roetti, Electronic Structure Theory: From Molecules to Crystals (in preparation)

${ }^{20}$ V. R. Saunders, in Methods in Computational Molecular Physics, edited by G. H. F. Diercksen and S. Wilson, 1 (Reidel, Dordrecht, Netherlands, 1984).

${ }^{21}$ J. M. André, L. Gouverneur and G. Leroy, Int. J. Quant. Chem. 1, 427 (1967); Int. J. Quant. Chem. 1, 451 (1967); J. M. André, J. Chem. Phys. 50, 1536 (1969).

${ }^{22}$ J. Delhalle, J. M. André, Ch. Demanet and J. L. Brédas, Chem. Phys. Lett. 54, 186 (1978).

${ }^{23}$ V. R. Saunders, C. Freyria-Fava, R. Dovesi, and C. Roetti, Comp. Phys. Comm. 84, 156 (1994).

${ }^{24}$ D. E. Parry, Surf. Science 49, 433 (1975); 54, 195 (1976) (Erratum).

${ }^{25}$ P. P. Ewald, Ann. Phys. (Leipzig) 64, 253 (1921).

${ }^{26}$ V. R. Saunders, C. Freyria-Fava, R. Dovesi, L. Salasco, and C. Roetti, Mol. Phys. 77, 629 (1992).

${ }^{27}$ L. E. McMurchie and E. R. Davidson, J. Comput. Phys. 26, 218 (1978). 
28 S. F. Boys, Proc. Roy. Soc. A 200, 542 (1950).

${ }^{29}$ R. McWeeny, Nature 166, 21 (1950).

${ }^{30}$ R. Dovesi, C. Pisani, C. Roetti, and V. R. Saunders, Phys. Rev. B 28, 5781 (1983).

31 T. Helgaker and P. R. Taylor, Theor. Chim. Acta 83, 177 (1992).

${ }^{32}$ F. E. Harris, in Theoretical Chemistry: Advances and Perspectives, Vol. 1, 147 (1975), edited by H. Eyring and D. Henderson, Academic Press, New York 


\section{TABLES}

TABLE I. CO molecule, all tolerances high enough that all the integrals are done without approximation. The carbon atom is placed at $(0 \AA, 0 \AA, 0 \AA)$, the oxygen atom at $(0.8 \AA, 0.5$ $\AA, 0.4 \AA$ ). To calculate the numerical force in $\mathrm{x}$-direction, the $\mathrm{x}$-coordinate of the oxygen atom is changed (column 1). The Hartree-Fock energy is displayed in column 2. The numerical force is determined from 2 points at $(0.8 \pm \delta) \AA$ and displayed in column 3 . The analytical derivative for $\mathrm{x}=0.8 \AA$ is displayed in column 4 . Basis sets of the size $[2 s 2 p 1 d]$ were used.

\begin{tabular}{cccc}
\hline \hline x-coordinate of oxygen & energy & $\begin{array}{c}\text { numerical derivative } \\
\text { (x-component) }\end{array}$ & $\begin{array}{c}\text { analytical derivative } \\
\text { (x-component) }\end{array}$ \\
$\AA$ & $E_{h}$ & $E_{h} / a_{B}$ & \\
0.799 & $-1.107642201574 \mathrm{E}+02$ & 0.376915 & \\
0.7999 & $-1.107648645627 \mathrm{E}+02$ & 0.376913 & \\
0.79999 & $-1.107649287000 \mathrm{E}+02$ & 0.376914 & \\
0.80000 & $-1.107649358230 \mathrm{E}+02$ & & \\
0.80001 & $-1.107649429453 \mathrm{E}+02$ & & \\
0.8001 & $-1.107650070153 \mathrm{E}+02$ & & \\
0.801 & $-1.107656446886 \mathrm{E}+02$ & & \\
\hline \hline
\end{tabular}


TABLE II. CO molecule, when varying the ITOL parameters. The first and second parameter should be reasonably high, the fourth and fifth should both be high or identical to obtain accurate forces.

\begin{tabular}{|c|c|c|}
\hline \multirow[t]{3}{*}{$I T O L$ values } & numerical derivative & analytical derivative \\
\hline & (x-component) & (x-component) \\
\hline & $E_{h} / a_{B}$ & $E_{h} / a_{B}$ \\
\hline 666612 & 0.37691 & 0.376913 \\
\hline 2020202020 & 0.37691 & 0.376913 \\
\hline 420202020 & 0.37691 & 0.376912 \\
\hline 320202020 & 0.37747 & 0.377389 \\
\hline 220202020 & 0.38883 & 0.388260 \\
\hline 202202020 & 0.37684 & 0.376821 \\
\hline 201202020 & -7.40676 & -1.246409 \\
\hline 202012020 & -8.09240 & -8.09240 \\
\hline 202020120 & -0.51905 & -3.504654 \\
\hline 202020201 & 0.40892 & 0.359847 \\
\hline 20202011 & -33.34823 & -33.348229 \\
\hline
\end{tabular}


TABLE III. CO molecule, arranged periodic in 0 dimensions ("molecule"), in 1 dimension ("polymer"), in 2 dimensions ("slab") and in 3 dimensions ("bulk"). We display the accuracy of the gradient as a function of the number of sampling points, and as a function of the ITOL parameters.

dimension number of sampling points ITOL parameters numerical derivative analytical derivative (x-component) (x-component)

\begin{tabular}{|c|c|c|c|c|}
\hline 0 & - & $\begin{array}{llllll}6 & 6 & 6 & 6 & 12\end{array}$ & 0.37691 & 0.376913 \\
\hline 1 & 5 & $\begin{array}{llllll}6 & 6 & 6 & 6 & 12\end{array}$ & 0.37659 & 0.376633 \\
\hline 1 & 5 & 6961212 & 0.37662 & 0.376647 \\
\hline 2 & 34 & $\begin{array}{llllll}6 & 6 & 6 & 6 & 12\end{array}$ & 0.37630 & 0.376335 \\
\hline 3 & 8 & 6666612 & 0.37571 & 0.375742 \\
\hline 3 & 260 & $\begin{array}{llllll}6 & 6 & 6 & 6 & 12\end{array}$ & 0.37565 & 0.375679 \\
\hline 3 & 260 & 6868814 & 0.37570 & 0.375720 \\
\hline 3 & 260 & 886814 & 0.37573 & 0.375721 \\
\hline
\end{tabular}


TABLE IV. MgO at a lattice constant of $4.21 \AA$. We compare numerical and analytical derivatives when moving the oxygen ion parallel to the $\mathrm{x}$-direction. Basis sets of the size $[3 s 2 p]$ were used.

\begin{tabular}{ccc}
\hline \hline displacement of oxygen & $\begin{array}{c}\text { analytical derivative } \\
(\text { x-component })\end{array}$ & $\begin{array}{c}\text { numerical derivative } \\
\text { in \% of lattice constant }\end{array}$ \\
+1 & $E_{h} / a_{B}$ & $E_{h} / a_{B}$ \\
+2 & -0.005923 & -0.00593 \\
+3 & -0.012254 & -0.01226 \\
\hline \hline
\end{tabular}

